\title{
Real-time Compensation for Angular-dependent Color Shift in TN LCDs Wayne Cheng
}

\author{
Department of Photonics and Display Institute, National Chiao Tung University, Hsinchu, Taiwan
}

\begin{abstract}
The angular-dependent color shift in a TN LCD is corrected at real-time by manipulating the input signals in an FPGA. The viewing direction of the user is captured by a video camera and face detection software.
\end{abstract}

\section{Introduction}

Limited viewing angle is the major drawback of twisted nematic (TN) LCDs. Not only the luminance contrast ratio, but also the color of a TN LCD shifts when viewed from a large angle. To cope with the angular-dependent color shift, a number of different approaches have been proposed in the past years. For instance, optically, the color shift can be reduced by design optimization of optical films and polarizers. Inside a pixel, the multiple-domain vertical alignment (MVA) design can widen the acceptable viewing angle. Similar solutions include patterned vertical alignment (PVA), advanced super view (ASV), and in-plane switching (IPS) modes. These approaches, unaware of user's position, widen the range of acceptable view angles, but sacrifice the color performance at a specific viewing angle.

The motivation of our work is to capture the user's position such that the luminance contrast ratio and color shift can be corrected at run-time by adjusting the gamma dynamically. We incorporate a video camera, which is a common accessory of portable computers, cell phones, and PDA, along with the face detection software. The user's viewing direction can be obtained once the face location is recognized. The gamma curve can then be adjusted at run-time based on pre-calculated lookup table, which is derived from characterization data from a ConoScope ${ }^{T M}$. In our prototype, we use a field programmable gate array (FPGA) board to intercept and manipulate the video signals. A computer and webcam are used to detect the user's face location. We also developed a viewing angle emulator to visualize the resulting images. The preliminary results affirm the feasibility of cameraassisted real-time angular-dependent color shift correction.

\section{Previous Works}

Marcu et al. proposed a method of compensating for the color shift at different locations [1]. Li et al. proposed an idea of dynamically compensating for the color shift according to the viewer's position [2]. These two approaches, focusing on display design, reduce the color shift based on the viewer's position. Moshnyaga et al. proposed using a video camera to monitor the user's attention such that the LCD can dim its backlight to conserve power consumption when the user looks away [3]. Our motivation is to combine these approaches to minimize the angular-dependent color shift.

\section{Angular-dependent Color Shift}

The conventional metric of evaluating the viewing angle of LCDs is the luminance contrast ratio. A viewing angle is acceptable if the luminance ratio of white to black, $L_{\max } / L_{\min }$, is no less than a certain threshold. Although simple and easy to apply, the luminance contrast ratio is insufficient to represent the color performance at large viewing angles. We have proposed an alternative metric in [4], which uses degradation of color saturation to measure acceptable viewing angles.

Our target system is a 19" Viewsonic VA912 TFT-LCD driven by an nVidia GeForce 6600-based 256MB graphics card in a WinTel computer. We used a ConoScope ${ }^{\mathrm{TM}}$ to measure the luminance and chromaticity from all possible angles within a hemisphere. The measurement sessions were repeated every 15 graylevels from 15 to 255. The measurement data were then exported to MS Excel for calculating color differences, data analysis and chart plotting. Fig. 1 shows the luminance measurement results on three different graylevels: 75,135 , and 195 . The upper three contour charts show the distributions of luminance variations. The horizontal crosssectional distributions (along the 9-to-3-o'clock plane) are shown in the lower three charts

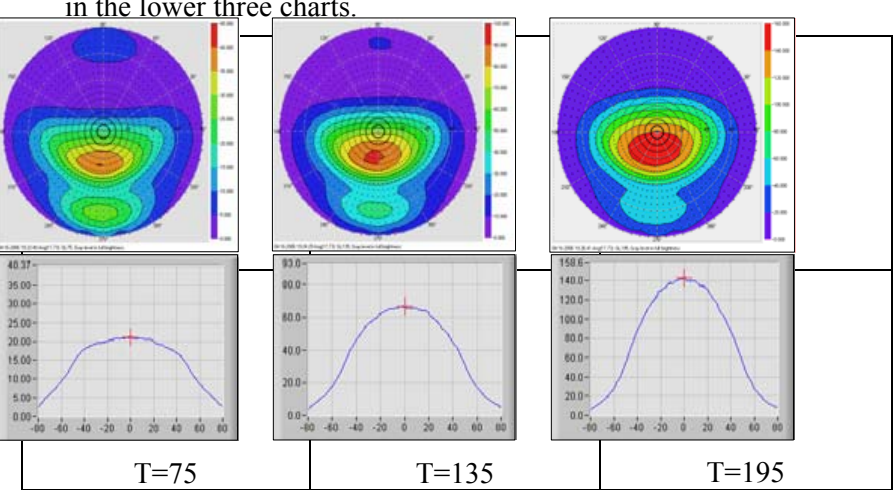

Fig. 1 Luminance measurements show angular dependency (upper) and graylevel dependency (lower).

The angular and graylevel dependency is so irregular that dynamic correction is inevitable. Fig. 2 shows the angular chromaticity differences on different graylevels. On graylevel 255 , the chromaticity difference near $0^{\circ}$ is very small but increases quickly with viewing angle. On the other hand, graylevel 135 has the biggest chromaticity difference near $0^{\circ}$ but decreases quickly with viewing angle.

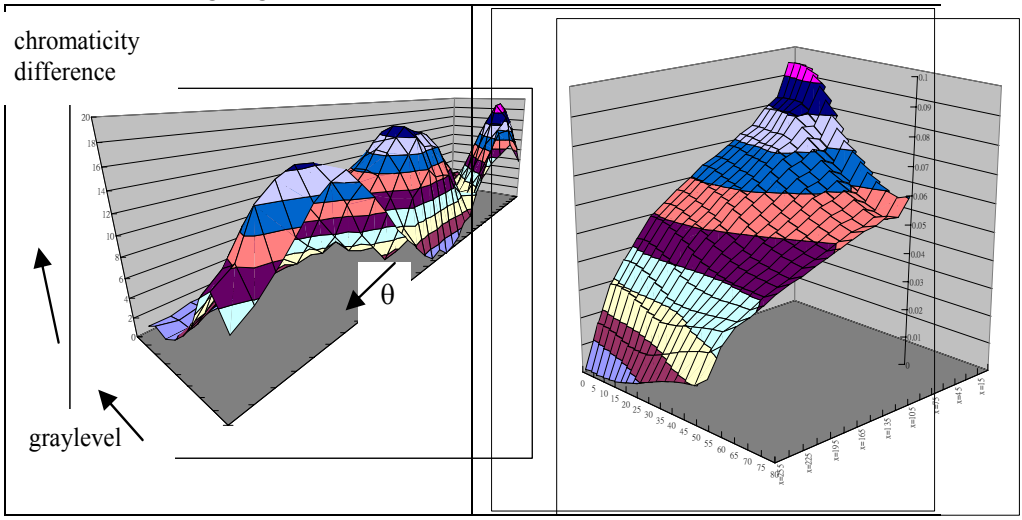

Fig. 2 (a) 3D-view of Fig. 1. (b) Average chromaticity

difference as a function of solid-angle (increasing in 2 o'clock direction) and graylevel (in 10 'clock direction). 
We also calculated the average chromaticity difference within a solid angle. The results are shown in Fig. $2 b$ as a function of solid-angle and graylevel. On each graylevel, the average chromaticity difference increases as the solid-angle enlarges. However, the increasing rates of the higher graylevels are steeper than those of the lower graylevels. Notice that for each solidangle, the chromaticity difference is monotonically decreasing with graylevel.

Given the characterization data, we calculated the inverse mapping of color shift and built a lookup table in terms of CIEXYZ coordinates, which was used by our prototype FPGA board and viewing angle emulator.

\section{Viewing Direction}

Face detection is a well-establish technique by the machine vision society. A number of free and open algorithms/implementations can be found on the Internet. We used Sebastien Marcel's Torch3 vision library [5].

To comprehend how users utilize viewing angles, we recorded observers' face movement while they were performing three requested tasks: game-playing, text-editing, web-surfing, and movie-watching. A sample of face tracing during game-playing is shown in Fig. 3, in which the irrelevant events such as coughing, adjusting glasses and scratching head were removed to simplify the data analysis. Although more experiments are to be conducted to collect data from more observers on different tasks, the preliminary findings show that the viewing angle variation can be modeled by a Gaussian distribution, whose standard deviation depends on the task in the way that game-playing $>$ text-editing $>$ web-surfing $>$ movie-watching.

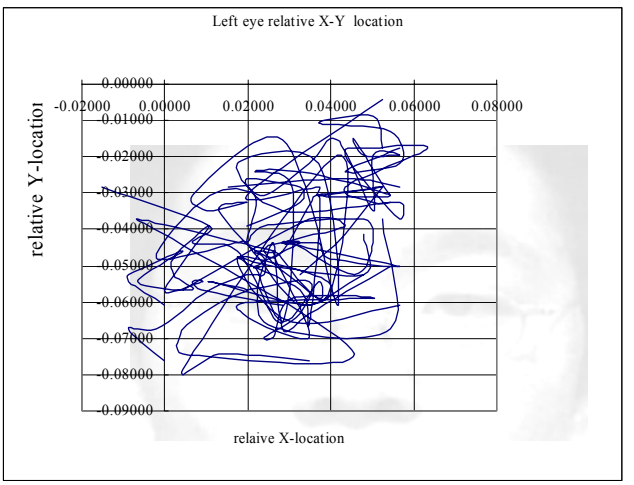

Fig. 3 Trace of face movement during a game-playing task.

\section{Real-time Color Correction}

The proposed algorithm was implemented in VHDL on an FPGA board, the Spartan3-based Xilinx Multimedia Development Board. This prototype receives NTSC composite video signal, processes the image, and outputs the VGA signal to drive the external 19" TFT-LCD monitor. The block diagram is shown in Fig. 4.

We have also developed a software tool that emulates the visual effect by using the given characterization data (cf. Fig. 5). The program takes a given image as well as the viewing angle, and then generates the simulated output image. The user specifies filename of the image of interest. The viewing angle $(\theta, \delta)$ can be entered as values or via a graphical interface. The program calculates the expected color shift by using table lookup and interpolation. It then shows the simulated images at real-time on the present display. A non-LCD monitor is required to avoid angular-dependent distortion. This tool reproduces the visual effect according to the characterization database. Particularly, the users can visualize the colorimetric performance of multiple LCDs simultaneously without the physical devices.

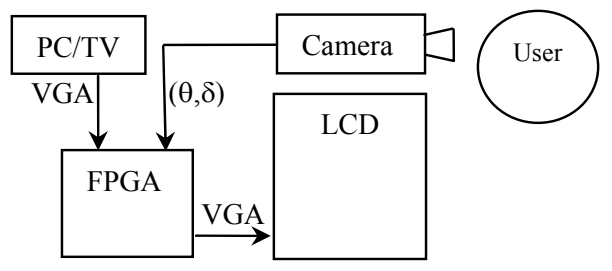

Fig. 4 Block diagram of the proposed system.

\section{Conclusions}

We employed the well-established face detection technique to obtain the user's viewing direction such that the angulardependent color shift can be corrected accordingly at run-time. The concept relies on the video camera and computing power for face detection. For mass production, the correction should be embedded in the gamma corrector. Future works include how to deal with multiple users/faces and how to apply the same concept to power (backlight) management.

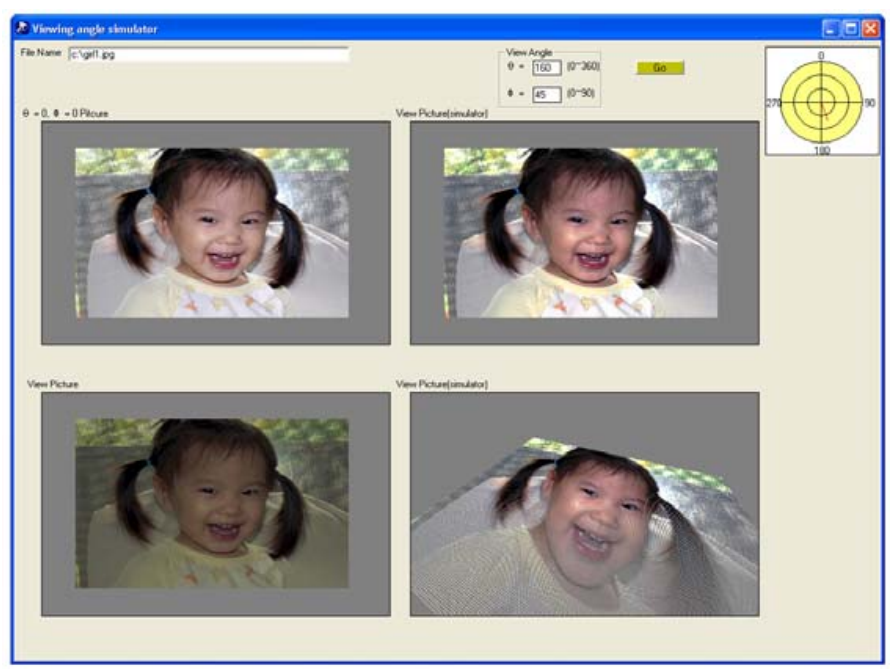

Fig. 5 Screen shot of the viewing angle emulator.

\section{REFERENCES}

[1] G. Marcu et al., "Color correction in TFTLCD Displays for compensation of color dependency with the viewing angle," SID 02 Digest, pp. 713-715.

[2] B. Li et al., "Adaptive display color correction based on realtime viewing angle estimation," SID 04 Digest, pp. 330-333.

[3] V. G. Moshnyaga et al., "LCD display energy reduction by user monitoring," International Computer CD 2005.

[4] S-F. F. Chen, W-C. Cheng, H-P. D. Shieh, "CSD: a new unified threshold metric of evaluating LCD viewing angle by color saturation degradation," IEEE/OSA Journal of Display Technology, 2006.

[5] http://www.idiap.ch/ marcel. 